\title{
Simulation of Furnace System with Uncertain Parameter
}

Wan Munirah W.M ${ }^{1}$, T. Ahmad ${ }^{2 *}$, S. Ahmad ${ }^{1}$ and A. Ashaari ${ }^{1}$

${ }^{1}$ Department of Mathematical Science, Faculty of Science, Universiti Teknologi Malaysia, 81310 UTM Johor Bahru, Johor, Malaysia. ${ }^{2}$ Ibnu Sina Institute for Fundamental Science, Nanotechnology Research Alliance, Universiti Teknologi Malaysia, 81310 UTM Johor Bahru, Johor, Malaysia

*Corresponding Author: tahir@ibnusina.utm.my

\section{Article history :}

Received 21 September 2014

Accepted 15 February 2015

\section{GRAPHICAL ABSTRACT}

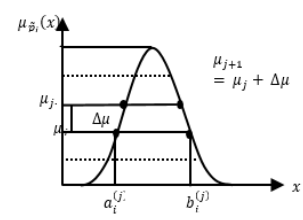

\section{ABSTRACT}

Energy system in power plants is one of the most frequently mentioned areas in thermal energy. The aim of this study is to model the heat transfer of a furnace in order to minimize energy losses. The furnace system is simulated using fuzzy arithmetic, namely, the uncertain model inputs are represented by fuzzy numbers. The analysis of the model is presented in this paper.

Keywords: Fuzzy arithmetic, Uncertain models, Furnace system

(C) 2015 Penerbit UTM Press. All rights reserved http://dx.doi.org/10.11113/mjfas.v1 $1 \mathrm{n} 1.334$

\section{INTRODUCTION}

Furnace is a thermal equipment in which water or air be heated to be circulated throughout a building in a heating system. Furnace is used in variety, for example household furnace is a major appliance that is installed to provide heat for house space. The most common fuel source for modern furnaces is natural gas others include coal, liquefied petroleum gas, wood or fuel oil.

In order to increase the furnace system efficiency, it is important to understand the physical phenomena of the combustion process in a furnace system. For example a furnace operating at $2400 \mathrm{~F}$ with $25 \%$ excess air could experience $24 \%$ fuel savings by reducing excess combustion air to $10 \%$ [1]. Through improvement of process efficiency, it will produce the quality product of better furnaces system. To improve the heating system from furnace, the main characteristic of furnace need to be studied on the basis of simulations of an appropriate mathematical model. Therefore, the need for new approaches in modeling is much influenced by competition, energy efficiency and the demand for low cost operation.

The parameters of the model are uncertain because they exhibit variability depending on the quantity of input. The model input that consist of the fuel flow, air flow and exhaust gas flow usually can be quantified with a high degree of imprecision. So, the best approach to solve this problem is by using fuzzy arithmetic in order to take into account the uncertainties in the furnace model.

The characteristic of the advanced fuzzy arithmetic is the elimination of restrictions in its area of applications. That is, it can be used to evaluate fuzzy rational expressions and also can be applied to simulate static or dynamic systems with fuzzy valued parameters [2].
In short, the uncertain model parameters and the model inputs are represented by fuzzy numbers where the membership function is characterized by Gaussian functions. As an abbreviated form, the notation is [2]

$$
\tilde{p}=g f n\left(\bar{x}, \sigma_{1}, \sigma_{r}\right)
$$

To define a Quasi-Gaussian fuzzy number $\tilde{p} \in \tilde{P}^{\prime}(R)$ with the membership function

$$
\mu_{\tilde{p}}(x)=\left\{\begin{array}{l}
\exp \left[-\frac{(x-\bar{x})}{2 \sigma_{1}^{2}}\right] \text { for } x<\bar{x} \\
\exp \left[-\frac{(x-\bar{x})}{2 \sigma_{r}^{2}}\right] \text { for } x \geq \bar{x}
\end{array} \quad \forall x \in R\right.
$$

The modal value is denoted by the parameter $\bar{x}$, and $\sigma_{1}$ and $\sigma_{r}$ denote the left-hand and right-hand spreads, corresponding to the standard deviations of the Gaussian distribution in (see Fig. 1).

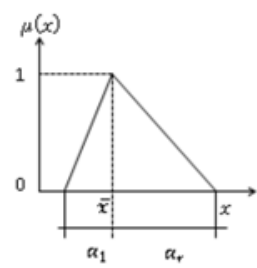

(a)

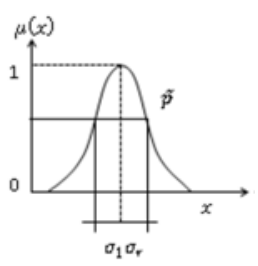

(b)
Figure 1. (a) Triangular fuzzy number (b) Gaussian fuzzy number.

\section{THE FURNACE MODEL}

The parameters used in the development of the model for furnace system are the input and the state parameters [3] presenting in Table 1.

Figure 2 shows the model of the boiler system. The effectiveness of this modelling approach is 
illustrated by implementing it in a furnace system of a combined cycle power plant, which is regarded as an important constituent in the heat system of a boiler.

Table 1. Parameters

\begin{tabular}{lll}
\hline Identifier & Unit & Definition \\
\hline Input : & & \\
\hline $\boldsymbol{w}_{\boldsymbol{F}}$ & $\mathrm{kg} / \mathrm{s}$ & Fuel flow to the furnace \\
$\boldsymbol{w}_{\boldsymbol{A}}$ & $\mathrm{kg} / \mathrm{s}$ & Air flow to the furnace \\
$\boldsymbol{w}_{\boldsymbol{G}}$ & $\mathrm{kg} / \mathrm{s}$ & Exhaust gas flow from the gas turbine \\
$\boldsymbol{h}_{\boldsymbol{G}}$ & $\mathrm{J} / \mathrm{kg}$ & Enthalpy of exhaust gas from the gas \\
& & turbine \\
$\boldsymbol{h}_{\boldsymbol{A}}$ & $\mathrm{J} / \mathrm{kg}$ & Inlet air enthalpy \\
$\boldsymbol{\theta}$ & $\mathrm{rad}$ & Tilt angle coefficient \\
$\boldsymbol{T}_{\boldsymbol{s t}}$ & $K$ & Temperature of superheater metal tubes \\
$\boldsymbol{T}_{\boldsymbol{r} h}$ & $K$ & Temperature of reheater metal tubes \\
$\boldsymbol{T}_{\boldsymbol{e t}}$ & $K$ & Temperature of economizer metal tubes \\
\hline State: & & \\
\hline $\boldsymbol{\rho}_{E G}$ & $\mathrm{Kg} / \mathrm{m}^{3}$ & Density of exhaust gas from the boiler \\
\hline
\end{tabular}

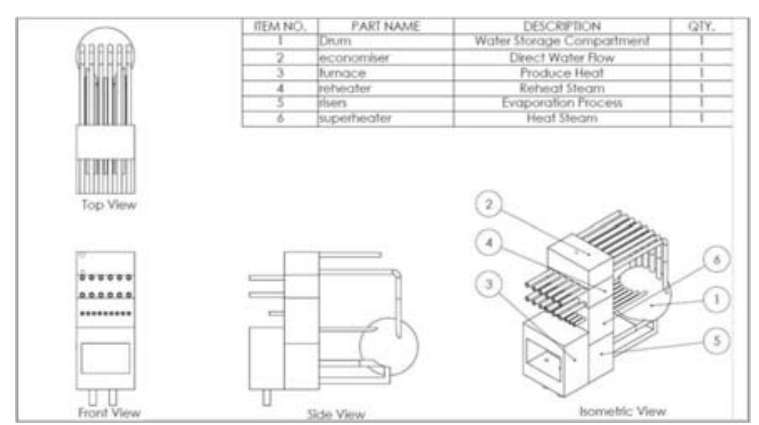

Figure 2. Model of Boiler System

The following is the model for the inflow of fuel, gas and air into the Furnaces System adopted from [3].

Exhaust gas flow through the boiler:

$W_{E G}=K_{F} P_{G}$

Furnace gas pressure:

$P_{g}=R_{E G} \rho_{E G} T_{g}$

Differential equation:

$\frac{d \rho_{E G}}{d t}=\frac{1}{V_{F}}\left(W_{F}+W_{A}+W_{G}-W_{E G}\right)$

State equation:

$$
\frac{d \rho_{E G}}{d t}=-\frac{K_{F} R_{E G} T_{g}}{V_{F}} \rho_{E G}+\frac{W_{F}}{V_{F}}+\frac{W_{A}}{V_{F}}+\frac{W_{G}}{V_{F}}
$$

\section{IMPLEMENTATION OF FUZZY ARITHMETIC}

Given in general a problem with a number of $n$ independent parameters which are assumed to be uncertain, the parameters can be represented by fuzzy numbers $\tilde{p}_{i}$ with $i=1,2, \ldots, n$. As illustrated in Fig. 3, each of the fuzzy numbers can be decomposed into a set
Pi of $m+1$ intervals $X_{i}^{(j)}, j=0,1, \ldots, \mathrm{m}$, of the form [4].

$$
P_{i}=\left\{X_{i}^{(0)}, X_{i}^{(1)}, \ldots, X_{i}^{(m)}\right\}
$$

$$
\begin{aligned}
X_{i}^{(j)} & =\left[a_{i}^{(j)}, b_{i}^{(j)}\right], a_{i}^{(j)} \leq b_{i}^{(j)}, \\
i & =1,2, \ldots, n, \quad j=0,1, \ldots, m .
\end{aligned}
$$

A fuzzy parameterized model is expected to show non-monotonic behavior with respect to $n$, for $n>1$ fuzzy value parameter $\tilde{p}_{i}$ with $i=1,2, \ldots, n$. Thus, the model is used general transformation method. The intervals $X_{i}^{(j)}, i=1,2, \ldots, n, j=0,1, \ldots, m-2$, are considered for the transformation scheme. The intervals are now transformed into arrays $\hat{X}_{i}^{(j)}$ of the form

$X_{i}^{(j)}=\underbrace{\left(\left(\gamma_{1, i}^{(j)}, \gamma_{2, i}^{(j)}, \ldots, \gamma_{(m+1-j),}^{(j)}\right), \ldots,\left(\gamma_{1, i}^{(j)} \gamma_{2, i}^{(j)}, \ldots, \gamma_{(m+1-j), i}^{(j)}\right)\right)}_{(m+1-j)^{i-j}(m+1-j)^{- \text {tuples }}}$

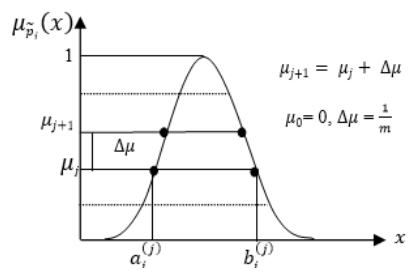

Figure 3. Implementation of a fuzzy number $\tilde{p}_{i}$ by decomposition into intervals.

$$
\begin{aligned}
& \gamma_{l, i}^{(j)}=\underbrace{\left(c_{l, i}^{(j)}, \ldots, c_{l, i}^{(j)}\right)}_{(m+1-j)^{n-i} \text { elements }} \\
& c_{l, i}^{(j)}=\left\{\begin{array}{cc}
a_{i}^{(j)} & \text { for } l=1 \text { and } j=0,1, \ldots, m, \\
\frac{1}{2}\left(c_{l-1, i}^{(j+1)}+c_{l, i}^{(j+1)}\right) & \text { for } l=2,3, \ldots, m-j \text { and } j=0,1, \ldots, m-2, \\
b_{i}^{(j)} & \text { for } l=m-j+1 \text { and } j=0,1, \ldots, m,
\end{array}\right.
\end{aligned}
$$

Assuming the problem to be given by the arithmetical expression $F$ in the form

$\tilde{q}=F\left(\tilde{p}_{1}, \tilde{p}_{2}, \ldots, \tilde{p}_{n}\right)$

Then, the evaluation is then carried out by evaluating the expression separately at each of the $2^{\mathrm{n}}$ positions of the fields using the conventional arithmetic for crisp numbers. The result of the problem can be expressed in its decomposed and transformed form by the fields $\hat{Z}^{(j)}, j=0,1, \ldots, m$, the $k$-th element ${ }^{\mathrm{k}} \hat{Z}^{(j)}$ of the array $\hat{Z}^{(j)}$ given by

$$
\begin{gathered}
{ }^{\mathrm{k}} \hat{Z}^{(j)}=F\left({ }^{\mathrm{k}} \hat{x}_{1}^{(j)},{ }^{\mathrm{k}} \hat{x}_{2}^{(j)}, \ldots,{ }^{\mathrm{k}} \hat{x}_{n}^{(j)}\right) \\
k=1,2, \ldots, 2^{n},
\end{gathered}
$$

Finally, the fuzzy-valued result $\tilde{q}$ of the problem can be achieved in its decomposed form 
$Z^{(j)}=\left[\mathrm{a}^{(\mathrm{j})}, \mathrm{b}^{(\mathrm{j})}\right], j=0,1, \ldots, m$

By retransforming the fields $\hat{Z}^{(j)}$ including a certain correction procedure according to the recursive formulas

$a^{(j)}=\min _{k}\left(a^{(j+1)},{ }^{\mathrm{k}} \hat{z}^{(j)}\right)$

$b^{(j)}=\max _{k}\left(b^{(j+1)},{ }^{\mathrm{k}} \hat{z}^{(j)}\right), j=0,1, \ldots, m-1$,

and

$a^{(m)}=\min _{k}\left({ }^{\mathrm{k}} \hat{\boldsymbol{Z}}^{(m)}\right)=\max _{k}\left({ }^{\mathrm{k}} \hat{\boldsymbol{Z}}^{(m)}\right)=b^{(m)}$

\section{ANALYSIS}

The problem with uncertainties can be achieved with the implementation of fuzzy arithmetic. The fuzzy valued result of the problem only shows the overall influence of all the uncertain parameters. Therefore, the degrees of influence of the different uncertain model parameters of a system on its output are certainly not equal in general. In this regard, one can determine the percentages to which the $n$ uncertain parameters of the system contribute to the overall uncertainty of the system output. Instead of reducing the array $\hat{Z}^{(j)}$ to the interval $Z^{(j)}$, as in the retransformation step of the transformation method, the supplementary information given by the values and the arrangement of the elements in $\hat{Z}^{(j)}$ can be used [5]. The coefficients for general transformation method are as follows [4].

The coefficients $\eta_{i}^{(j)}, i=1,2, \ldots, n, j=0$, $1, \ldots, m-1$, are to be determined for the general transformation method:

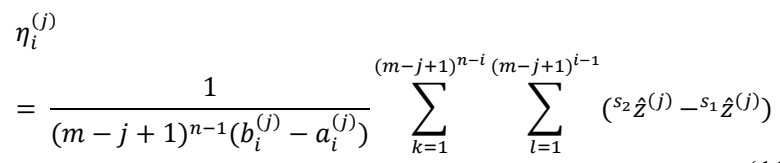

with

$s_{1}(k, l)=k+(l-1)(m-j+1)^{n-i+1}$

and

$s_{2}(k, l)=k+[(m-j+1) l-1](m-j+1)^{n-i}$

The values $a_{i}^{(j)}$ and $b_{i}^{(j)}$ denote the lower and upper bound of the interval $X_{i}^{(j)}$, and ${ }^{\mathrm{k}} \hat{Z}^{(j)}$ is the $k^{\text {th }}$ elements of the array $\hat{Z}^{(j)}$. The coefficients $\eta_{i}^{(j)}$ can be interpreted as gain factors which express the effect of the uncertainty of the $i^{\text {th }}$ parameter on the uncertainty of the output $z$ of the problem at the membership level $\mu_{j}$. To become independent of a specific level of membership, some mean gain factors $\eta_{i}^{(j)}$ are computed such that

$$
\bar{\eta}_{i}=\frac{\sum_{j=1}^{m-1} \mu_{j} \eta_{i}^{(j)}}{\sum_{j=1}^{m-1} \mu_{j}}
$$

Finally, the degree of influence, the normalized values $\rho_{i}$ can be determined for $i=1,2, \ldots, n$, using

$\rho_{i}=\frac{\sum_{j=1}^{m-1} \mu_{j}\left|\eta_{i}^{(j)}\left(a_{i}^{(j)}+b_{i}^{(j)}\right)\right|}{\sum_{q=1}^{n} \sum_{j=1}^{m-1} \mu_{j}\left|\eta_{q}^{(j)}\left(a_{q}^{(j)}+b_{q}^{(j)}\right)\right|}$

which satisfies the condition

$\sum_{i=1}^{n} \rho_{i}=1$

Thus, the values $\rho_{i}$ quantify the influence of the $i^{\text {th }}$ varying parameter $\tilde{\rho}_{i}$ on the overall variation $\tilde{q}$ of the problem output $z$, assuming that every parameter to be varied relatively to the same extent.

\section{IMPLEMENTATION}

For the simulation of the model, the parameter $w_{F}, w_{A}, w_{G}, h_{G}, h_{A}, \boldsymbol{\theta}, T_{s t}, T_{r h}$, and $T_{e t}$ are considered as independent parameters, represented by quasi Gaussian fuzzy numbers $\tilde{p}_{i}$ of the form

$\tilde{p}=g f n\left(\bar{x}, \sigma_{1}, \sigma_{r}\right), \quad i=1,2, \ldots, n$,

The value $\bar{x}_{i}$ of the fuzzy parameters is listed in Table 2 , and the standard deviation $\sigma_{i}$ is defined as

$\sigma_{i}=2 \% \bar{x}_{i}, \quad i=1,2, \ldots, n$,

for each parameter, corresponding to a worst-case deviation of $\pm 30 \%$.

The non-monotonic behaviour is likely to appear as a consequence of linear elements in the model equations (6). The uncertain furnace model is simulated and analysed using an appropriate form of the general transformation method.

In contrast to the universally valid general form [2], the transformation method can be applied in its extended form to reduce the computational costs of the simulation. The analysis of the furnace model shows that upon evaluating the classification criterion, only eight out of nine modal parameters can be regarded as type-g-parameters and others are type-r-parameters. Hence the extended transformation method can be applied by setting $\bar{n}=8$.

Table 2. Modal values and standard deviation of the uncertain model parameters.

\begin{tabular}{cccc}
\hline Parameter & $\overline{\boldsymbol{x}}$ & $\boldsymbol{\sigma}$ & Dimension \\
\hline$\widetilde{\boldsymbol{p}}_{\mathbf{1}}=\boldsymbol{w}_{\boldsymbol{F}}$ & 14.1 & 0.1 & $\mathrm{~kg} / \mathrm{s}$ \\
$\widetilde{\boldsymbol{p}}_{\mathbf{2}}=\boldsymbol{w}_{\boldsymbol{A}}$ & 64.09 & 0.02 & $\mathrm{~kg} / \mathrm{s}$ \\
$\widetilde{\boldsymbol{p}}_{\mathbf{3}}=\boldsymbol{w}_{\boldsymbol{G}}$ & 23.18 & 0.06 & $\mathrm{~kg} / \mathrm{s}$ \\
$\widetilde{\boldsymbol{p}}_{\mathbf{4}}=\boldsymbol{h}_{\boldsymbol{G}}$ & $6.9 \times 10^{5}$ & 0.05 & $\mathrm{~J} / \mathrm{kg}$ \\
$\widetilde{\boldsymbol{p}}_{\mathbf{5}}=\boldsymbol{h}_{\boldsymbol{A}}$ & $2.5 \times 10^{5}$ & 0.07 & $\mathrm{~J} / \mathrm{kg}$ \\
$\widetilde{\boldsymbol{p}}_{\mathbf{6}}=\boldsymbol{\theta}$ & 0.88 & 0.3 & $\mathrm{rad}$ \\
$\widetilde{\boldsymbol{p}}_{\mathbf{7}}=\boldsymbol{T}_{\boldsymbol{s t}}$ & 736.97 & 0.04 & $\mathrm{~K}$ \\
$\widetilde{\boldsymbol{p}}_{\mathbf{8}}=\boldsymbol{T}_{\boldsymbol{r} \boldsymbol{h}}$ & 743.71 & 0.07 & $\mathrm{~K}$ \\
$\widetilde{\boldsymbol{p}}_{\mathbf{9}}=\boldsymbol{T}_{\boldsymbol{e t}}$ & 412.54 & 0.05 & $\mathrm{~K}$ \\
\hline
\end{tabular}


As a result of the simulation of the model with a decomposition number $\mathrm{m}=5$, the uncertain furnace model $\tilde{q}=\tilde{\rho}_{E G}$ can be obtained as the fuzzy-valued output of the model. It is plotted against time in Fig. 4, featuring contour lines for the membership levels $\mu=$ 0.0 to $\mu=1.0$ in steps of $\Delta \mu=0.2$.

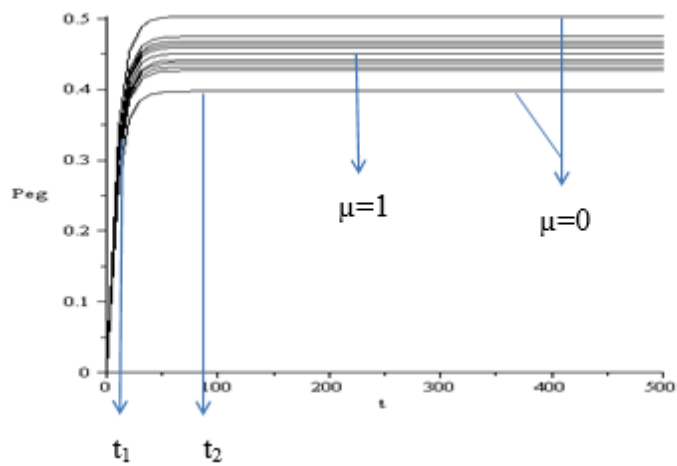

Figure 4. Contour plot of the uncertain furnace model $\tilde{\rho}_{E G}(t)$ with the membership grade $\mu$ as contour parameter in steps of $\Delta \mu=0.2$.

The value of $\tilde{\rho}_{E G}(t)$ is increased at the beginning and relatively constant over a long period of time. The two points at time, $t_{1}=20 \mathrm{~s}$ and $t_{2}=80 \mathrm{~s}$ are taken. The worst case range of the uncertainty amount is 0.1 $\mathrm{kg} / \mathrm{m}^{3}$ at $t_{1}$ and $0.105 \mathrm{~kg} / \mathrm{m}^{3}$ at $t_{2}$.

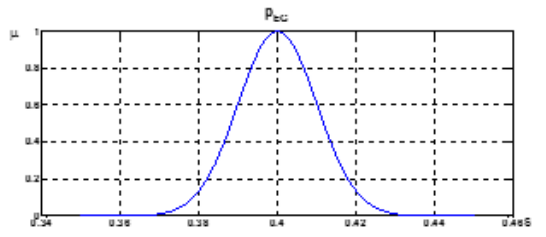

(a)

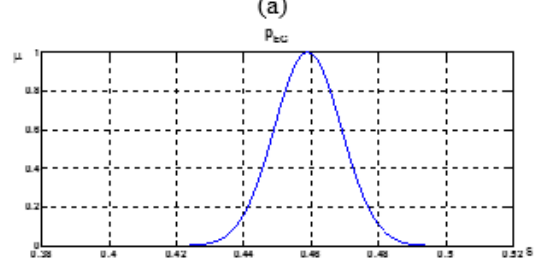

(b)

Figure 5. Membership functions of a) the uncertain furnace model $\tilde{\rho}_{E G}\left(t_{1}\right)$ at $t_{1}=20 \mathrm{~s}$ and $\left.\mathrm{b}\right)$ the uncertain furnace model $\tilde{\rho}_{E G}\left(t_{2}\right)$ at $t_{2}=80 \mathrm{~s}$.

Fuzzy-valued $\tilde{\rho}_{E G}\left(t_{1}\right)$ and $\tilde{\rho}_{E G}\left(t_{2}\right)$ show significantly strong variation in shape to the original symmetric quasi-Gaussian shape of the uncertain model parameters $\tilde{p}_{1}, \tilde{p}_{2}, \ldots, \tilde{p}_{9}$ in Fig.5. One can conclude that the linear elements in the model only have a slightly effect on the furnace system under the given operating condition.

From the evaluation of the degree of influence $\rho_{i}(\mathrm{t}), \mathrm{i}=1,2, \ldots, 9$, only three out of nine uncertain model parameters show an observable influence on the outflow of furnaces. Additionally, only the degrees of influence $\rho_{i}(\mathrm{t})$ for the parameters $w_{F}, w_{A}$ and $w_{G}$ exhibit non-zero values. The influence of the remaining parameter $h_{G}, h_{A}, \theta, T_{s t}, T_{r h}$, and $T_{e t}$ can be neglected. The following are the degree of influence $\rho_{1}, \rho_{2}$, and $\rho_{3}$.

$$
\begin{aligned}
& \rho_{1}=7.55 \% \\
& \rho_{2}=68.61 \% \\
& \rho_{3}=23.84 \%
\end{aligned}
$$

The model parameter of $\rho_{2}$ show high degree of influence, while the parameter of $\rho_{1}$ and $\rho_{2}$ have only a moderate impact on the model (see Fig. 6).

Based on Fig. 6 (b) the density of exhaust gas is decrease when the air is excessive [1]. This effect is due to increasing of heat and reducing the exhaust gas at the same time. Additionally, the parameter $\tilde{p}_{2}=w_{A}$ shows significant impact to $\tilde{\rho}_{E G}(t)$ compare to the others.

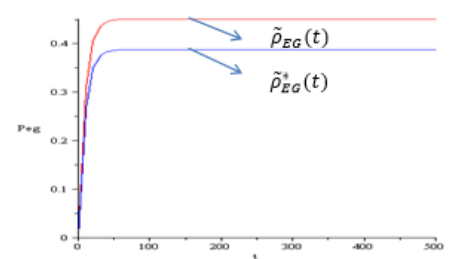

(a)

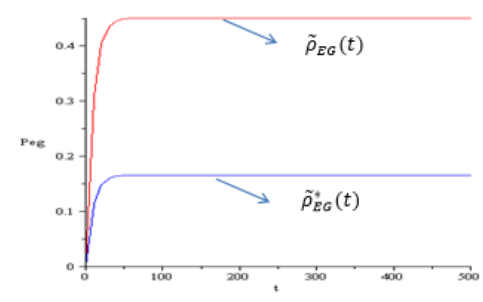

(b)

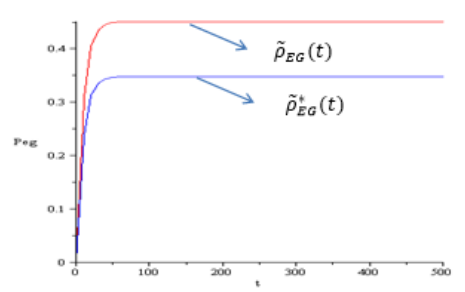

(c)

Figure 6. Density of exhaust gas $\tilde{\rho}_{E G}(t)$, and Estimated density of exhaust gas $\tilde{\rho}_{E G}^{*}(t)$ with the value of (a) $\rho_{1}=7.55 \%$ (b) $\rho_{2}=68.61 \%$ (c) $\rho_{3}=23.84 \%$.

\section{CONCLUSIONS}

The application of fuzzy arithmetic to simulate the furnace model proves a very successful approach. The evolution of the air flow to the furnace is significantly affected by uncertainties in the parameter compare to the fuel flow of the furnace by those related to the combustion process. Hence, to improve the furnace efficiency, efforts should focus on the quantification of the air flow parameters than the exact quantification of the fuel flow of the combustion process. 


\section{ACKNOWLEDGEMENTS}

These works are supported by Ibnu Sina Institute, My Phd Scholarship from Ministry of Higher Education, Malaysia and University Teknologi Malaysia.

\section{REFERENCES}

[1] Morris HA (2007) Advanced Modeling for Small Glass Furnaces, Master of Science in Mechanical Engineering, Department of Mechanical Engineering Morgantown.
[2] M. Hanss, (2005) Applied Fuzzy Arithmetic An Introduction with Engineering Applications, Springer.

[3] Ordys, A.W., Pike, A.W., Johnson, M.A., Katebi, R.M. and Grimble, M.J., Modelling and Simulation of Power Generation Plants. London : Springer-Verlag, 1994.

[4] M. Hanss, "The transformation method for the simulation and analysis of systems with uncertain parameters," vol. 130, pp. 277-289, 2002.

[5] M. Hanss, O. Nehls, M. Hanss, and O. N. De, "Simulation of the Human Glucose Metabolism Using Fuzzy Arithmetic," pp. 1-05, 2000 . 